\title{
The changing milliarcsecond radio morphology of the gamma-ray binary LS 5039
}

\author{
M. Ribó ${ }^{1}$, J. M. Paredes ${ }^{1}$, J. Moldón ${ }^{1}$, J. Martí $^{2}$, and M. Massi ${ }^{3}$ \\ 1 Departament d'Astronomia i Meteorologia, Universitat de Barcelona, Martí i Franquès 1, 08028 Barcelona, Spain \\ e-mail: [mribo;jmoldon]@am.ub.es; jmparedes@ub.edu \\ 2 Departamento de Física (EPS), Universidad de Jaén, Campus Las Lagunillas s/n, 23071 Jaén, Spain \\ Max Planck Institut für Radioastronomie, Auf dem Hügel 69, 53121 Bonn, Germany
}

Received 1 August 2007 / Accepted 21 December 2007

ABSTRACT

\begin{abstract}
Context. LS 5039 is one of the few TeV emitting X-ray binaries detected so far. The powering source of its multiwavelength emission can be accretion in a microquasar scenario or wind interaction in a young nonaccreting pulsar scenario.

Aims. To present new high-resolution radio images and compare them with the expected behavior in the different scenarios.

Methods. We analyze Very Long Baseline Array (VLBA) radio observations that provide morphological and astrometric information at milliarcsecond scales.

Results. We detect a changing morphology between two images obtained five days apart. In both runs there is a core component with a constant flux density, and an elongated emission with a position angle (PA) that changes by $12 \pm 3^{\circ}$ between both runs. The source is nearly symmetric in the first run and asymmetric in the second one. The astrometric results are not conclusive.

Conclusions. A simple and shockless microquasar scenario cannot easily explain the observed changes in morphology. An interpretation within the young nonaccreting pulsar scenario requires the inclination of the binary system to be very close to the upper limit imposed by the absence of X-ray eclipses.
\end{abstract}

Key words. stars: individual: LS 5039 - X-rays: binaries - radio continuum: stars - radiation mechanism: non-thermal

\section{Introduction}

Very high energy (VHE) gamma-ray emission in the TeV range has been detected in four massive X-ray binaries. PSR 1259-63 contains a young nonaccreting millisecond (ms) radio pulsar (Aharonian et al. 2005a). The accreting/ejecting microquasar Cygnus X-1 has recently been found to be a TeV emitter (Albert et al. 2007). LS I +61 303 (Albert et al. 2006), suggested to be a fast precessing microquasar (Massi et al. 2004), displays a changing milliarcsecond radio morphology that Dhawan et al. (2006) interpreted in the context of the interaction between the wind of the companion and the relativistic wind of a young nonaccreting ms pulsar (Dubus 2006). Finally, the nature of the powering source in LS 5039, whether accretion or rotation, is unknown (see Aharonian et al. 2005b, 2006, for the TeV results).

The system LS 5039 contains a compact object of unknown nature, with mass between 1.4 and $5 M_{\odot}$, orbiting every 3.9 days an ON6.5 V((f)) donor (Casares et al. 2005). The detection of elongated asymmetric emission in high-resolution radio images obtained with the Very Long Baseline Array (VLBA) and the European VLBI Network (EVN) was interpreted as evidence of its microquasar nature, and suggested that the source was persistently producing mildly relativistic ejections with a velocity of $\sim 0.15 c$ (Paredes et al. 2000, 2002). Although the X-ray spectra are compatible with those of accreting black holes during the so-called low/hard state (Bosch-Ramon et al. 2005), the radio spectra are optically thin with a spectral index of -0.5 (Martí et al. 1998; Ribó et al. 1999). Theoretical modelling in the microquasar scenario has allowed researchers to reproduce the observed spectral energy distribution (SED) from radio to VHE gamma-rays (Paredes et al. 2006). However, the lack of clear accretion signatures and the similarities with the SEDs of PSR 1259-63 and LS I +61 303 has led other authors to model its multiwavelength emission using the scenario of wind interactions (Dubus 2006). One of the predictions of this kind of modelling is the periodic change in the direction and shape of the extended radio morphology as well as in the peak position of the radio core, depending on the orbital phase.

In this paper, we report on new high-resolution radio images of LS 5039 in an attempt to disentangle the two possible scenarios using morphological and astrometric information.

\section{Observations}

We observed LS 5039 with the National Radio Astronomy Observatory (NRAO) VLBA and the Very Large Array (VLA) at $5 \mathrm{GHz}$ frequency on 2000 June 3 and 8 . We used the VLA in its $\mathrm{C}$ configuration, both as a connected interferometer and as a phased array. The two observing sessions, hereafter run A and run B, spanned from 4:30 to 12:30 UT on the corresponding dates, and were thus centered on MJD 51698.4 and MJD 51703.4, respectively. The orbital phases of the system were in the range $0.43-0.51$ for run $\mathrm{A}$ and in the range $0.71-0.79$ of the following orbital cycle for run B (using the ephemeris from Casares et al. 2005).

We performed the observations using the phase-referencing technique, switching between the phase reference calibrator J1825-1718 and LS 5039, separated 2.47, with cycling times of $5.5 \mathrm{~min}$, compatible with the expected coherence time. The fringe finder was 3C 345. The ICRF source J1911-2006, located at 11.9 from LS 5039, was observed every 22 min to monitor the performance of the observations. To check the stability of the 
astrometry we observed the source $\mathrm{J} 1837-1532$, located at 3.38 from the phase reference calibrator J1825-1718.

We recorded the data with 2-bit sampling at $256 \mathrm{Mbps}$, at left-hand circular polarization. A total bandwidth of $64 \mathrm{MHz}$ was provided by 8 sub-bands. The data were processed at the VLBA correlator in Socorro, using an integration time of $4 \mathrm{~s}$.

The position used hereafter for the phase reference source J1825-1718, obtained by means of dedicated geodetic Very Long Baseline Interferometry (VLBI) observations, is $\alpha_{\mathrm{J} 2000.0}=$ $18^{\mathrm{h}} 25^{\mathrm{m}} 36.53237 \pm 0.00021$ (or \pm 3.0 mas) and $\delta_{\mathrm{J} 2000.0}=$ $-17^{\circ} 18^{\prime} 49^{\prime} .8534 \pm 00^{\prime} 0045$ (or \pm 4.5 mas) in the frame of ICRFExt.1 (Craig Walker, private communication). However, this information was not available at the time of correlation, which was performed for a calibrator position shifted by $\Delta \alpha=+57.0$ mas and $\Delta \delta=+21.4$ mas. In the case of LS 5039, due to its proper motion (see Ribó et al. 2002), the source was found to be $\Delta \alpha=+7.6$ mas and $\Delta \delta=-16.2$ mas away from the correlated phase center, located at $\alpha_{\mathrm{J} 2000.0}=18^{\mathrm{h}} 26^{\mathrm{m}} 15^{\mathrm{s}} .05600$ and $\delta_{\mathrm{J} 2000.0}=-14^{\circ} 50^{\prime} 54^{\prime \prime} 2400$ (from Martí et al. 1998).

Regarding the VLA observations, the source 3C 286 was used for flux density calibration, and the source J1825-1718 for phase calibration and to phase up the array.

\section{Data reduction}

We performed the post-correlation data reduction using the Astronomical Image Processing System (AIPS) software package, developed and maintained by NRAO. The phased VLA position had to be corrected by $43 \mathrm{~cm}$, according to later geodetic measurements. The positions of J1825-1718 and LS 5039 were corrected using the task CLCOR. As recommended for phasereferencing experiments, we applied ionospheric and Earth Orientation Parameters corrections to the visibility data using the task CLCOR. A priori visibility amplitude calibration was done using the antenna gains and the system temperatures measured at each station. We then used the fringe finder to calibrate the instrumental phase and delay offsets. The fringe fitting (FRING) of the residual delays and fringe rates was performed for all the radio sources. We found very good solutions for 3C 345 , J1911-2006 and J1825-1718. Fringes for 15\% and 25\% of the baselines were missing for LS 5039 and J1837-1532, respectively. Typical data inspection and flagging were performed. The obtained self-calibrated images of J1911-2006, which show a one-sided jet, are very similar to those present in the VLBA calibrator source catalog, assessing the reliability of the observations and the initial data reduction.

For each run, we performed standard phase and amplitude self-calibration and phase self-calibration steps on LS 5039. As a compromise between angular resolution and sensitivity, we used a weighting scheme with robust 0 to produce the images with the task IMAGR. The obtained image had a synthesized beam of $\sim 6.4 \times 2.2$ mas in position angle (PA) of $\sim 0^{\circ}$. Extended emission was visible for both runs. To enhance the small-scale morphology, we produced final images using a convolving beam of $3.4 \times$ 1.2 mas in PA of $0^{\circ}$ (similar to what would be obtained by using robust -2 within AIPS or a uniform weighting scheme within DIFMAP). This convolving beam is the one we used previously in Paredes et al. (2000), allowing for a straightforward comparison of the images.

To measure the position of LS 5039 in both runs we used the phase-referencing technique, and transferred the phases of the calibrator J1825-1718, fitted with the task FRING, to our target source. The peak flux density of this extragalactic phasereference calibrator is $118 \mathrm{mJy} \mathrm{beam}^{-1}$ at $5 \mathrm{GHz}$, enough to provide useful phase information. However, it suffers scattering and becomes resolved with the VLBA, providing relatively noisy phase information on the longest baselines. We inspected and screened the phase delays and delay rates of J1825-1718. Tropospheric corrections were unsuccessful because the observation strategy was not adequate to account for them. Phasereferenced images of the target source with a synthesized beam of $\sim 4.9 \times 1.5$ mas in PA $\sim 0^{\circ}$ were obtained, and the peak position was measured with the AIPS task JMFIT.

\section{Results}

\subsection{VLA}

The VLA data of LS 5039 were compatible with a point-like source for the obtained synthesized beam of $5.5 \times 3.7^{\prime \prime}$ in PA of $\sim-4^{\circ}$. We measured the flux density of the source every $30 \mathrm{~min}$ and obtained a mean of $29.4 \mathrm{mJy}$ with a standard deviation of $\sigma=1.1 \mathrm{mJy}$ during the $8 \mathrm{~h}$ of run A, and $28.4 \mathrm{mJy}$ with $\sigma=$ $0.7 \mathrm{mJy}$ for run B. Therefore, LS 5039 displayed flux density variability below $4 \%$ at the $1 \sigma$ level within each 8 -h run. Similar results were obtained within 4-h halves. This guarantees that we can produce reliable VLBA images for each of the entire observation periods, as well as for 4-h intervals, provided the morphology does not change within such intervals.

\subsection{VLBA}

We show the final VLBA+phased VLA self-calibrated images in Fig. 1. The image obtained for run A displays a central core and bipolar and nearly symmetric extended emission with $\mathrm{PA} \simeq 116 \pm 2^{\circ}$, with enhanced emission toward the southeast. The total flux density recovered by the VLBA, obtained with the task TVSTAT within AIPS, is $\sim 25 \mathrm{mJy}$, representing $\sim 85 \%$ of the VLA value. The peak flux density of the core is $10.5 \mathrm{mJy}$ beam $^{-1}$. The image is similar to the one obtained with the same array in 1999 May 8, corresponding to orbital phases $0.12-0.15$, which showed a slightly more asymmetric extended emission in PA $\sim 125^{\circ}$, as can be seen in Paredes et al. (2000). In contrast, the image obtained for run B displays a core and bipolar but clearly asymmetric structure, with $\mathrm{PA} \simeq 128 \pm$ $2^{\circ}$ and enhanced emission toward the northwest. The total flux density recovered is $\sim 24 \mathrm{mJy}$, or $\sim 85 \%$ of the VLA value. The peak flux density of the core is $10.5 \mathrm{mJy} \mathrm{beam}^{-1}$, as in run A.

To characterize the extended emission we used UVFIT and JMFIT within AIPS, as well as model fitting tools within DIFMAP to check the reliability of the obtained results. The preferred model for run A data consisted of three Gaussian components to account for the core (Core1), the southeast (SE1) and the northwest (NW1) components. A similar model could fit the data for run $\mathrm{B}$, although the southeast (SE2) component is marginally fitted in this case. The fitted parameters are quoted in Table 1. As can be seen, the peak flux density of each component is $\sim 50 \%$ of its total flux density, although this is partially due to the convolving beam size being smaller than the synthesized beam size. In particular, we stress that the core components are not point-like.

Finally, we splitted the $8 \mathrm{~h}$ of data in each run into 4-h data sets. For each of them, we performed a similar calibration process as the one described above, and we modelled the final visibilities of the self-calibrated data. We measured no significant morphological differences between the two halves in any of the two runs. In particular, the distance between the fitted components Core 1 and SE1 is stable in $4 \mathrm{~h}$ within the errors 

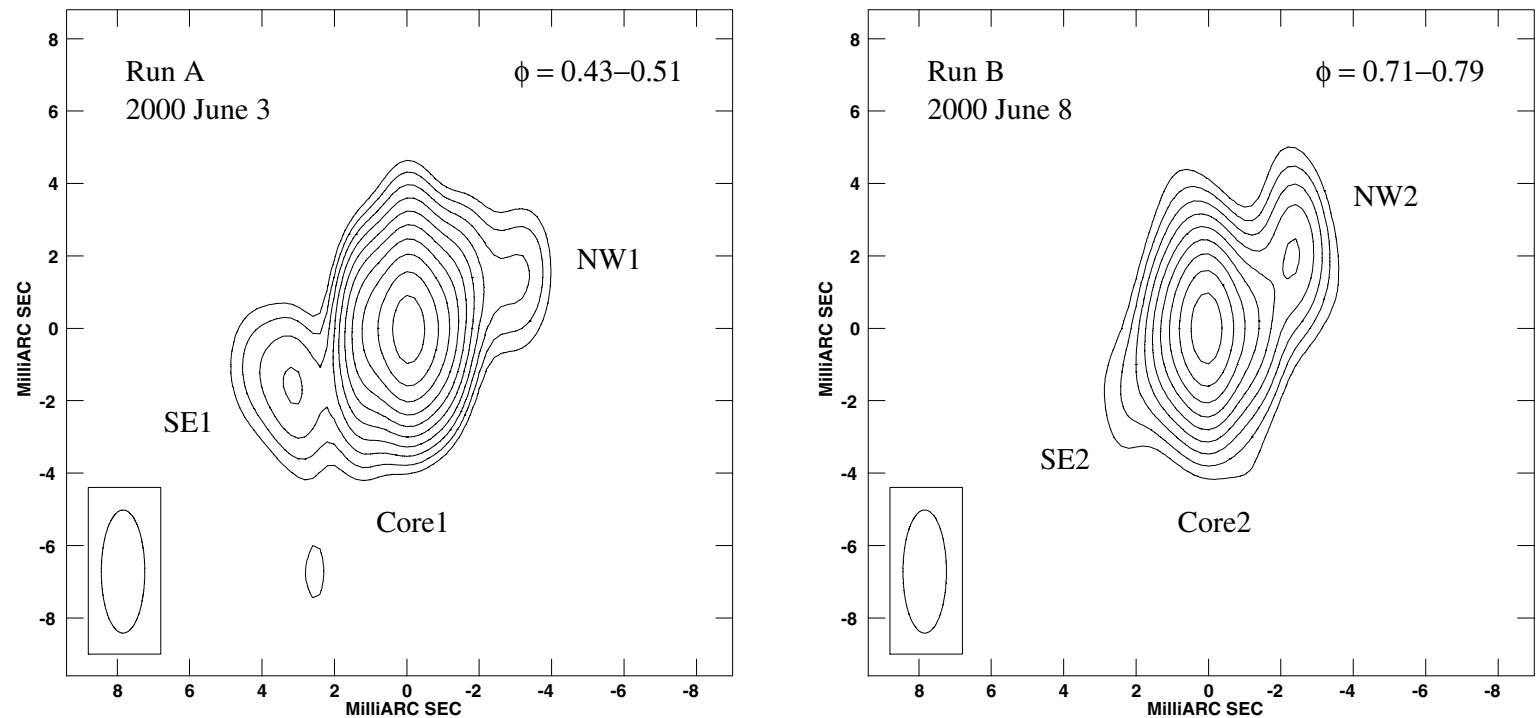

Fig. 1. VLBA+phased VLA self-calibrated images of LS 5039 at $5 \mathrm{GHz}$ obtained on 2000 June 3 (left) and 8 (right). North is up and east is to the left. Axes units are mas, and the $(0,0)$ position corresponds to the source peak in each image. The convolving beam, plotted in the lower left corner, has a size of $3.4 \times 1.2$ mas in PA of $0^{\circ}$. The first contour corresponds to 5 times the rms noise of the image $\left(0.08\right.$ and $0.11 \mathrm{mJy}^{\mathrm{beam}}{ }^{-1}$ for run $\mathrm{A}$ and $\mathrm{B}$, respectively), while consecutive ones scale with $2^{1 / 2}$. The dates and orbital phases are quoted in the images. There is extended radio emission that appears nearly symmetric for run A and clearly asymmetric for run B, with a small change of $\sim 10^{\circ}$ in its position angle. We have labeled the components fitted to the data, the parameters of which are listed in Table 1.

Table 1. Parameters of the Gaussian components fitted to the data for each 8-h run. Columns 3 and 4 list the peak and integrated flux densities of each component. Columns 5 to 8 list the polar and Cartesian coordinates of the components with respect to the peak position. Columns 9 to 11 list

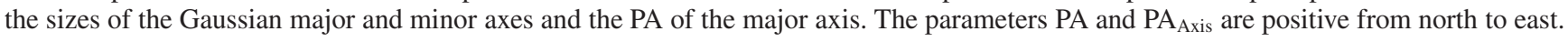

\begin{tabular}{|c|c|c|c|c|c|c|c|c|c|c|}
\hline$\overline{\text { Run }}$ & Comp. & $\begin{array}{c}\text { Peak } S_{5 \mathrm{GHz}} \\
{\left[\mathrm{mJy} \mathrm{beam}^{-1} \text { ] }\right.}\end{array}$ & $\begin{array}{l}S_{5 \mathrm{GHz}} \\
{[\mathrm{mJy}]}\end{array}$ & $\begin{array}{c}r \\
{[\mathrm{mas}]}\end{array}$ & $\begin{array}{l}\text { PA } \\
{\left[{ }^{\circ}\right]}\end{array}$ & $\begin{array}{c}\Delta \alpha \\
{[\mathrm{mas}]}\end{array}$ & $\begin{array}{c}\Delta \delta \\
{[\mathrm{mas}]}\end{array}$ & $\begin{array}{c}\text { Maj. Axis } \\
{[\mathrm{mas}]}\end{array}$ & $\begin{array}{c}\text { Min. Axis } \\
\text { [mas] }\end{array}$ & $\begin{array}{c}\mathrm{PA}_{\text {Axis }} \\
{\left[{ }^{\circ}\right]}\end{array}$ \\
\hline \multirow[t]{3}{*}{$\mathrm{A}$} & Core1 & $10.54 \pm 0.08$ & $20.0 \pm 0.2$ & $\overline{-}$ & 1 & - & - & $3.69 \pm 0.03$ & $2.09 \pm 0.02$ & $103 \pm 1$ \\
\hline & SE1 & $1.11 \pm 0.08$ & $2.6 \pm 0.2$ & $3.67 \pm 0.08$ & $115.9 \pm 1.7$ & $3.30 \pm 0.07$ & $-1.60 \pm 0.12$ & $4.1 \pm 0.3$ & $2.36 \pm 0.16$ & $17 \pm 5$ \\
\hline & NW1 & $0.88 \pm 0.08$ & $1.5 \pm 0.2$ & $3.29 \pm 0.09$ & $-63 \pm 2$ & $-2.92 \pm 0.08$ & $1.52 \pm 0.14$ & $3.6 \pm 0.3$ & $1.99 \pm 0.18$ & $3 \pm 6$ \\
\hline \multirow[t]{3}{*}{$\mathrm{B}$} & Core2 & $10.45 \pm 0.11$ & $17.6 \pm 0.3$ & - & - & - & - & $3.71 \pm 0.04$ & $1.8 \pm 0.2$ & $180 \pm 1$ \\
\hline & SE2 & $0.75 \pm 0.11$ & $1.8 \pm 0.4$ & $2.8 \pm 0.2$ & $129 \pm 5$ & $2.17 \pm 0.13$ & $-1.8 \pm 0.3$ & $4.6 \pm 0.7$ & $2.1 \pm 0.3$ & $1 \pm 7$ \\
\hline & NW2 & $2.22 \pm 0.11$ & $3.9 \pm 0.3$ & $2.94 \pm 0.06$ & $-52.2 \pm 1.4$ & $-2.32 \pm 0.04$ & $1.80 \pm 0.09$ & $4.3 \pm 0.2$ & $1.68 \pm 0.08$ & $174 \pm 2$ \\
\hline
\end{tabular}

$\left(\sigma_{\alpha}^{\mathrm{A}}=0.31 \mathrm{mas}, \sigma_{\delta}^{\mathrm{A}}=0.62 \mathrm{mas}\right)$. This is also the case between Core 2 and NW2 components $\left(\sigma_{\alpha}^{\mathrm{B}}=0.55\right.$ mas, $\sigma_{\delta}^{\mathrm{B}}=0.25$ mas $)$.

\subsection{Astrometry}

The phase-referenced image of LS 5039 obtained for run A, not shown here, displays a compact source with peak flux density of $6.7 \mathrm{mJy} \mathrm{beam}^{-1}$, and a faint extended emission toward the southwest $\left(\sim 2 \mathrm{mJy}\right.$ beam $^{-1}$; the rms is $\left.0.3 \mathrm{mJy} \mathrm{beam}^{-1}\right)$. The measured position of LS 5039 for run $\mathrm{A}$ is $\alpha_{\mathrm{J} 2000.0}=$ $18^{\mathrm{h}} 26^{\mathrm{m}} 15^{\mathrm{s}} .05653 \pm 0.00001$ (or $\left.\pm 0.15 \mathrm{mas}\right)$, and $\delta_{\mathrm{J} 2000.0}=$ $-14^{\circ} 50^{\prime} 54^{\prime \prime} .2564 \pm 0^{\prime}$. 0015 (or \pm 1.5 mas) (see discussion below). We recall that the reference coordinates are those of J1825-1718 listed in Sect. 2, which have systematic uncertainties of \pm 3.0 mas and \pm 4.5 mas, respectively. For checking purposes we splitted the data in blocks of 4- and 1-h lengths, made images for each block, and measured the corresponding positions. The peak position of LS 5039 appears to move $\Delta \alpha=+0.1 \pm$ 0.1 mas, $\Delta \delta=+2.8 \pm 0.2$ mas between the two 4-h blocks, and slightly more in the 1 -h blocks. The observed direction has a PA of $1.7 \pm 1.7^{\circ}$, which is the same as the line joining the positions of the source and the phase-reference calibrator J1825-1718. On the other hand, the expected error for differential astrometry is given by the separation $d$ in degrees from the phase-reference source and the offset $\Delta$ of its correlated position, according to $\Delta \times(d / 180) \times \pi$ (see Walker 1999). Plugging our offset of 60.9 mas and a distance of 2.47 we obtain an error of 2.6 mas, very similar to the observed displacement of LS 5039. A similar procedure using 4-h blocks for the astrometric check source J1837-1532 $(d=3.38)$ reveals motion of the peak of this extended source by $3.2 \pm 0.4$ mas at PA $=46 \pm 8^{\circ}$, as expected. Therefore, these secular motions appear to be purely instrumental. The errors assigned to the coordinates of LS 5039 quoted above are half of the total secular motion measured in 1-h blocks.

The phase-referenced image of run B, not shown here, reveals a double source in the eastwest direction. The two components have similar peak flux densities of $3.6 \pm 0.1 \mathrm{mJy} \mathrm{beam}^{-1}$, much lower than the one of the self-calibrated core, and are separated $2.5 \pm 0.1$ mas in PA of $87 \pm 5^{\circ}$. Moreover, 4 - and 1 -h blocks reveal a fading of the western component and a brightening of the eastern one along the run (as well as a similar secular motion as in run A). This symmetric double structure is in contrast to the asymmetric structure seen in the self-calibrated image shown in Fig. 1-right. Tropospheric errors, which affect the phase-referenced image and cannot be accounted for, can easily split Core 2 into the observed double source. Therefore, the precise position of the peak of LS 5039 cannot be measured in run B. 


\section{Discussion}

The observations of LS 5039 reported here, obtained with the VLBA on two runs separated by 5 days, show a changing morphology at mas scales. In both runs there is a core component with a constant flux density within errors, and elongated emission with a PA that changes by $12 \pm 3^{\circ}$ between both runs. The source is nearly symmetric in run A and asymmetric in run B (see Fig. 1).

In the microquasar scenario, and assuming ballistic motions of adiabatically expanding plasma clouds without shocks (see Mirabel \& Rodríguez 1999), the morphology of run A can be interpreted as a double-sided jet emanating from a central core with the southeast component as the approaching one. The relative distances of the components to the core would imply a bulk motion with velocity $\beta>0.06 \pm 0.02$, whereas the flux asymmetry implies $\beta>0.08 \pm 0.02$ for discrete ejections. The angle $\theta$ between the jet and the line of sight is restricted to $<87^{\circ}$. These results are similar to those previously found with VLBA and EVN images (Paredes et al. 2000, 2002). We can compute, for different $\theta$ angles, the expected displacement of the SE1 component with respect to Core 1 in $4 \mathrm{~h}$ using the measured value for the flux asymmetry of $\beta \cos \theta=0.08$, and compare it to the upper limits for this displacement quoted at the end of Sect. 4.2. This provides a constraint of $\theta<48^{\circ}$ at the $3 \sigma$ level. In contrast, in run $\mathrm{B}$ the northwest component appears to be the approaching one emanating from the core. The distances from Core 2 to the components NW2 and SE2 are very similar and do not imply any significant relativistic motion. The flux asymmetry implies $\beta>0.11 \pm 0.03$ and $\theta<84^{\circ}$ for discrete ejections. As for run $\mathrm{A}$, our measured upper limits of any displacement in $4 \mathrm{~h} \mathrm{im-}$ ply $\theta<45^{\circ}$ at the $3 \sigma$ level. In conclusion, the lack of proper motions implies that, for the measured flux asymmetries, the SE1 jet should be pointing at $\theta<48^{\circ}$, and the NW2 jet at $\theta<45^{\circ}$. In this context, jet precession is needed to explain this behavior. If the precession axis is close to the plane of the sky, as in SS 433 (Blundell \& Bowler 2004), the precession cone should have a semi-opening angle $>45^{\circ}$ to fulfill the $\theta$ limits quoted above. If the precession axis is close to the line of sight, a small precession of a few degrees could explain the images of runs A and B. However, in both cases the PA of the jet should vary considerably, in contrast to the small range covered by all observed values at mas scale, between 115 and $140^{\circ}$ (Paredes et al. 2000, 2002).

Alternatively, the morphology detected in run B could be the result of a discrete ejection with core suppression where Core 2 is the approaching component and NW2 the receding one, while there is no radio emission at the origin of the ejection. In this case, the flux asymmetry implies $\beta>0.21 \pm 0.01$ and $\theta<78 \pm$ $1^{\circ}$. The measured upper limits of the relative motion of these components in $4 \mathrm{~h}$ provide a strong constraint of $\theta<14^{\circ}$ at the $3 \sigma$ level. Imposing $\beta \cos \theta=0.21$, the origin of the discrete ejection (i.e., the position of the binary system or the position of Core 1 in run A) should be placed at $\Delta \alpha=-1.4$ mas and $\Delta \delta=+1.1$ mas from Core 2 in Fig. 1 . Although this would be self-consistent if the precise position of Core 2 corresponds to the east component of the phase-referenced image of run $\mathrm{B}$, it could be a chance coincidence produced by tropospheric errors. One should also invoke jet precession on short timescales to explain the change in PA of the extended emission. However, large $\mathrm{X}$-ray and radio flux density variations are observed in microquasars displaying discrete ejections (see Fender 2006, and references therein), while the peak and total radio flux densities of LS 5039 are strikingly constant (see also, Ribó et al. 1999;
Clark et al. 2001), and there is no evidence of an X-ray flare in 11.5 years of $R X T E / A S M$ data.

In the young nonaccreting pulsar scenario, the different morphologies we have detected at different orbital phases could be due to the change of the relative positions between the pulsar and the companion star along the orbit (see Dubus 2006, for details). However, observations at different orbital phases have always revealed a very similar PA for the extended emission, which can only be observed if the binary system is seen nearly edge on. On the other hand, the absence of X-ray eclipses places an upper limit of $i \lesssim 75^{\circ}$ in this scenario (Dubus 2006). Therefore, these two restrictions imply an inclination angle that should be close to the upper limit of $75^{\circ}$. We note that this is much higher than the inclination angle if the binary system is pseudo-synchronized (Casares et al. 2005).

In conclusion, a simple and shockless microquasar scenario cannot easily explain the observed changes in morphology. On the other hand, an interpretation within the young nonaccreting pulsar scenario requires the inclination of the binary system to be very close to the upper limit imposed by the absence of X-ray eclipses. Precise phase-referenced VLBI observations covering a whole orbital cycle are necessary to trace possible periodic displacements of the peak position, expected in this last scenario, and to obtain morphological information along the orbit. These will ultimately reveal the nature of the powering source in this gamma-ray binary.

Acknowledgements. We thank an anonymous referee for useful comments that helped to improve this paper. We are grateful to Simon Garrington for allowing us to schedule dedicated MERLIN observations of possible phase-reference sources. We are grateful to Craig Walker for his advice in the use of possible calibrators. The position of J1825-1718 was provided by observations from the joint NASA/USNO/NRAO geodetic/astrometric observing program. We thank Bob Campbell, Eduardo Ros and José Carlos Guirado for useful discussions about the phase-referencing problems. The NRAO is a facility of the National Science Foundation operated under cooperative agreement by Associated Universities, Inc. Part of the data reduction was done at JIVE with the support of the European Community - Access to Research Infrastructure action of the Improving Human Potential Programme, under contract No. HPRI-CT-1999-00045. This research has made use of the NASA's Astrophysics Data System Abstract Service, and of the SIMBAD database, operated at CDS, Strasbourg, France. M.R., J.M.P., and J.M. acknowledge support by DGI of the Spanish Ministerio de Educación y Ciencia (MEC) under grants AYA2007-68034-C03-01 and AYA2007-68034C03-02 and FEDER funds. M.R. acknowledges financial support from MEC through a Ramón y Cajal fellowship. J.M. is also supported by Plan Andaluz de Investigación of Junta de Andalucía as research group FQM322.

\section{References}

Aharonian, F., Akhperjanian, A. G., Aye, K.-M., et al. 2005a, A\&A, 442, 1 Aharonian, F., Akhperjanian, A. G., Aye, K.-M., et al. 2005b, Science, 309, 746 Aharonian, F., Akhperjanian, A. G., Bazer-Bachi, A. R., et al. 2006, A\&A, 460, 743

Albert, J., Aliu, E., Anderhub, H., et al. 2006, Science, 312, 1771

Albert, J., Aliu, E., Anderhub, H., et al. 2007, ApJ, 665, L51

Blundell, K. M., \& Bowler, M. G. 2004, ApJ, 616, L159

Bosch-Ramon, V., Paredes, J. M., Ribó, M., et al. 2005, ApJ, 628, 388

Casares, J., Ribó, M., Ribas, I., et al. 2005, MNRAS, 364, 899

Clark, J. S., Reig, P., Goodwin, S. P., et al. 2001, A\&A, 376, 476

Dhawan, V., Mioduszewski, A., \& Rupen, M. 2006, PoS, Proceedings of the VI

Microquasar Workshop: Microquasars and Beyond, ed. T. Belloni, 52.1

Dubus, G. 2006, A\&A, 456, 801

Fender, R. P. 2006, in Compact Stellar X-Ray Sources, ed. W. H. G. Lewin, \&

M. van der Klis (Cambridge University Press) [arXiv: astro-ph/0303339] Martí, J., Paredes, J. M., \& Ribó, M. 1998, A\&A, 338, L71

Massi, M., Ribó, M., Paredes, J. M., et al. 2004, A\&A, 414, L1

Mirabel, I. F., \& Rodríguez, L. F. 1999, ARA\&A, 37, 409

Paredes, J. M., Martí, J., Ribó, M., \& Massi, M. 2000, Science, 288, 2340

Paredes, J. M., Ribó, M., Ros, E., Martí, J., \& Massi, M. 2002, A\&A, 393, L99

Paredes, J. M., Bosch-Ramon, V., \& Romero, G. E. 2006, A\&A, 451, 259

Ribó, M., Reig, P., Martí, J., \& Paredes, J. M. 1999, A\&A, 347, 518

Ribó, M., Paredes, J. M., Romero, G. E., et al. 2002, A\&A, 384, 954

Walker, R. C. 1999, ASP Conf. Ser., 180, 433 